Visión Antataura

Vol.4, No.1: 36-51

Junio - Noviembre, 2020

Panamá

ISSN 2520-9892

\title{
Prevalencia y factores asociados al consumo de bebidas alcohólicas en estudiantes del distrito de Las Tablas
}

\section{Prevalence and factors associated with alcoholic beverages consumption in students of Las Tablas district}

\author{
Calixto Barrera $M^{1}$, Reiner Vergara ${ }^{2}$, Daniela Domínguez ${ }^{3}$, Alexandra González \\ Dianys Cedeño ${ }^{5}$, Daylin Solís ${ }^{6}$
}

\author{
${ }^{1}$ Postgrado en Docencia Superior; Profesor de Biología del Ministerio de Educación, Colegio Manuel María \\ Tejada Roca; calixto1207@gmail.com \\ ${ }^{2}$ Estudiante del Duodécimo grado del bachiller en Ciencias, año lectivo 2018, Colegio Manuel María Tejada \\ Roca; reinerveragara@outlook.com \\ ${ }^{3}$ Estudiantes del Duodécimo grado del bachiller en Ciencias, año lectivo 2018, Colegio Manuel María Tejada \\ Roca; dominguezdaniela12323@gmail.com \\ ${ }^{4}$ Estudiantes del Duodécimo grado del bachiller en Ciencias, año lectivo 2018, Colegio Manuel María Tejada \\ Roca; 25alexamichelle@gmail.com \\ ${ }^{5}$ Estudiantes del Duodécimo grado del bachiller en Ciencias, año lectivo 2018, Colegio Manuel María Tejada \\ Roca; dianyscs24@gmail.com \\ ${ }^{6}$ Estudiantes del Duodécimo grado del bachiller en Ciencias, año lectivo 2018, Colegio Manuel María Tejada \\ Roca; daylinsolis0404@gmail.com
}

Resumen: Con la finalidad de establecer la prevalencia y factores motivacionales asociados al consumo de bebidas alcohólicas, en estudiantes del colegio Manuel María Tejada Roca, se seleccionó una muestra de 139 estudiantes de octavo grado y 167 estudiantes de undécimo grado para aplicarles una encuesta de 20 ítems y la prueba AUDIT. Se obtiene una prevalencia de consumo de bebidas alcohólicas en octavo grado y undécimo grado, de $27,3 \%$ y $61,7 \%$ de los estudiantes, respectivamente, siendo en ambos niveles académicos superior en el sexo masculino. La edad promedio del primer consumo en undécimo grado fue 14,3 años, disminuyendo en octavo grado a 12,1 años. Un $56,7 \%$ de los encuestados señalan la curiosidad como principal factor que los motivó a consumir bebidas alcohólicas, destacándose las cervezas como favoritas, con 42,6 \% de las respuestas. Urgen programas preventivos al consumo de bebidas alcohólicas en estudiantes, ya que la prueba AUDIT indica que solo el 55,8 \% tiene consumo de bajo riesgo, mientras que 37,5 \% tiene un consumo riesgoso, $3,8 \%$ consumo perjudicial y $2,9 \%$ dependencia al alcohol. Se registra un rendimiento académico significativamente superior en estudiantes que no han consumido bebidas alcohólicas versus aquellos que si han consumido.

Palabras clave: consumo de bebidas alcohólicas, adolescentes, estudiantes, prueba AUDIT.

Abstract: In order to establish the prevalence and motivational factors associated with the consumption of alcoholic drinks in students of Manuel María Tejada Roca High School, a sample of 139 students of 8th grade and 167 students of 11th grade was selected for them to answer a survey of 20 Questions and the AUDIT Test. The prevalence of consumption of alcoholic drinks in 8th graders and 11th graders, reaches $27.3 \%$ and 61.7 $\%$ respectively, in students with higher consumption in males in both academic grades. The average age of the first consumption in 11th graders was 14.3 years; decreasing to 12.1 years in 8th graders. $56.7 \%$ of respondents pointed out being curiosity the main factor that motivated them to consume alcoholic drinks, 
standing out beers as the favorite ones, with $42.6 \%$ of the responses. Preventive programs for the in adolescents' consumption of alcoholic drinks are urgently needed, since the AUDIT Test indicates just only $55.8 \%$ have low risk consumption, meanwhile $37.5 \%$ have risky consumption, $3.8 \%$ harmful consumption and $2.9 \%$ alcohol addiction. A Significantly higher academic performance was recorded in students who had not consumed alcoholic drinks versus those who had.

Key words: alcoholic beverage consumption, adolescent, students, AUDIT test.

\section{Introducción}

En términos generales, consumir bebidas alcohólicas (alcohol) es una actividad socialmente aceptada, que suele iniciarse y establecerse en la adolescencia (Casas y Parrado, 2013; Chacón, 2013), etapa donde es frecuente desarrollar conductas de riesgo, entre ellas consumir alcohol y tabaco (Moniño et al., 2013). Siendo el alcohol una de las drogas más consumidas en la sociedad, su consumo es un creciente y preocupante problema social, debido a su alto grado de adicción (López - Cisneros et al., 2013).

El consumo de bebidas alcohólicas entre los adolescentes es una problemática de salud pública a nivel mundial (Cabrera - Jiménez et al., 2013; Flores, 2016), que provoca que su crecimiento y desarrollo físico, psicológico y académico se vea afectado negativamente, incluso interrumpido (Téllez y Cote, 2006). Además, lo predispone a las relaciones sexuales no planificadas, consumo de otras drogas y enfermedades físicas (Delgado et al., 2005), así como a embarazos no deseados y/o contagio de infecciones de transmisión sexual (D. M. González, comunicación personal, 3 de septiembre de 2018).

En los adolescentes, factores familiares y sociales influyen en el consumo de bebidas alcohólicas (Flores, 2016), así como la curiosidad (Espada, Méndez e Hidalgo, 2000). En otras palabras, los adolescentes aprenden a consumir bebidas alcohólicas en el hogar, estando la historia familiar de consumo de bebidas alcohólicas correlacionadas significativamente con el consumo de esta sustancia en adolescentes (Chacón, 2013; Landero y Villarreal, 2007). Este consumo se inicia a temprana edad al socializar con padres y amigos, continuando en fiesta y con amigos, por gusto y alegría (Landero y Villarreal, 2007; Morales- González et al., 2015), constituyéndose como una acción que facilita las relaciones sociales, además de ser un instrumento de cohesión entre el grupo de iguales y un elemento básico para la diversión (Pastor, 2002). Cabe destacar que, de manera general, los adolescentes consumen bebidas 
alcohólicas con mayor frecuencia y en mayor cantidad a medida que van creciendo (Delgado, Flores y Palos, 2007).

A nivel regional, Panamá, es el segundo país de Centroamérica con mayor consumo de bebidas alcohólicas per cápita (Simmons, 2016), lo que queda evidenciado en la tendencia de acompañar todas las celebraciones con consumo de bebidas alcohólicas (Domínguez, 2017; Torrijos, 2019). Al respecto, Chacón (2013) sostiene que más de la mitad de los adolescentes en Panamá, entre 15 y 19 años, saben qué es una resaca y que cada día son más los que prueban su primer trago, situación que obedece a la alta disponibilidad, bajo precio y publicidad constante de las bebidas alcohólicas (Monteiro, citado en Simmons, 2016), así como factores familiares, aspectos culturales y normativas que no se cumplen (Guzmán, citado en Domínguez, 2017).

Para el caso específico, de la provincia de Los Santos, investigación realizada por McDonald et al. (2015) en alumnos de octavo grado a nivel nacional, la ubica en primer lugar referente a la prevalencia de consumo de bebidas alcohólica. Esto puede deberse al hecho de residir en una provincia donde hay fiestas de calle todos los días, a la no regulación de estas actividades y la falta de aplicación de legislación que impida el acceso de menores de edad a estos eventos (D. M. González, comunicación personal, 3 de septiembre de 2018). Aunado, que Panamá carezca de políticas públicas que permitan contrarrestar la adicción al consumo de esta sustancia (Simmons, 2016), supone una grave problemática a corto y mediano plazo.

En base a la problemática expuesta, la investigación se realizó con el objetivo de establecer la prevalencia y factores motivacionales asociados al consumo de bebidas alcohólicas en estudiantes del colegio Manuel María Tejada Roca, a fin de establecer una línea base de conocimientos que permita al cuerpo directivo del colegio, profesores consejeros y departamento de orientación, autoridades del Ministerio de Educación y del MINSA, implementar programas que contribuyan a prevenir y/o disminuir el consumo de bebidas alcohólicas en adolescentes. Un segundo objetivo fue verificar estadísticamente si existen diferencias significativas en el rendimiento académico de estudiantes que consumen bebidas alcohólicas versus aquellos que no lo hacen. 


\section{Metodología}

En los meses de julio y agosto de 2018 se realizó un estudio descriptivo y transversal, en estudiantes de octavo grado (285 estudiantes) y undécimo grado (294 estudiantes), del colegio Manuel María Tejada Roca, distrito de Las Tablas, provincia de Los Santos. La muestra representó al menos un $48 \%$ de los estudiantes por nivel académico, y fue calculada según fórmula sugerida por Aguilar- Barojas (2005), (Anexo 1) con un índice de confiabilidad del $95 \%$ y un margen de error del 5\%. Para octavo grado estuvo conformada por 139 estudiantes (72 hombres y 77 mujeres), mientras que en undécimo grado fue de 167 estudiantes (75 hombres y 92 mujeres).

La autorización del estudio se logró mediante solicitud por escrito a la dirección del colegio. El muestreo fue aleatorio por conglomerado, utilizando el aula como unidad de selección. El mismo incluyó a estudiantes presentes al momento del estudio, que decidieran participar voluntariamente, inscritos en un solo nivel académico y residentes del distrito de Las Tablas. Esta investigación no atenta con la susceptibilidad y dignidad de los estudiantes encuestados, pues su participación fue voluntaria y se les aclaró que la información vertida era anónima y se utilizaría únicamente para fines de investigación.

Para la recolección de la información se utilizaron dos instrumentos. El primero, una encuesta de 20 ítems que contenía preguntas como: sexo, edad, edad del primer consumo, motivo inicial de consumo, persona con quien inició el consumo, tipo de bebida consumida regularmente, entre otras. Para evaluar el riesgo de consumo de alcohol durante los últimos 12 meses, se utilizó un segundo instrumento conocido como el Test de Identificación de los Trastornos Debidos al Consumo de Alcohol (AUDIT), el cual consta de 10 ítems que clasifican el consumo de alcohol en patrones de consumo; consumo de bajo riesgo de cero a siete puntos, consumo de riesgo de ocho a 15 puntos, consumo perjudicial de 16 a 19 puntos y dependencia al alcohol igual o mayor a 20 puntos (Babor, Higgins-Biddle, Saunders y Monteiro, 2001).

Los datos fueron analizados en el programa Microsoft Excel 2010; se obtuvieron estadísticas descriptivas como frecuencias y porcentajes, medidas de tendencia central como la media, y se aplicó el análisis de varianza de un factor, para verificar nuestras 
hipótesis de trabajo: "la edad promedio del primer consumo de bebidas alcohólicas es significativamente menor en estudiantes de octavo grado en comparación a estudiantes de undécimo grado" y "el rendimiento académico en estudiantes que han consumido bebidas alcohólicas es significativamente inferior en comparación a aquellos que no han consumido bebidas alcohólicas".

\section{Resultados y discusión}

El promedio de edad de los encuestados en octavo grado y undécimo grado fue de 13,5 años y 16,5 años, respectivamente, encontrándose que 141 (46,1\%) estudiantes han consumido bebidas alcohólicas, con una mayor prevalencia en varones. En este sentido, en octavo grado el $27,3 \%$ de los estudiantes (35,5 \% de los hombres y el $20,8 \%$ de las mujeres) han iniciado el consumo de bebidas alcohólicas, mientras que la prevalencia en undécimo grado se incrementa al $61,7 \%$ de los estudiantes $(69,3 \%$ de los hombres y $55,4 \%$ de las mujeres) (figura 1). Los datos obtenidos indican que, a mayor edad, la prevalencia de consumo de bebidas alcohólicas aumenta, independientemente del sexo, patrón que concuerda con lo descrito por Espada et al. (2000) para la región de Elche Alicante, España y lo registrado por Delgado et al. (2007) para el Distrito Federal, México.

Figura 1. Prevalencia del consumo de bebidas alcohólicas en estudiantes del distrito de Las Tablas, por sexo y nivel académico.

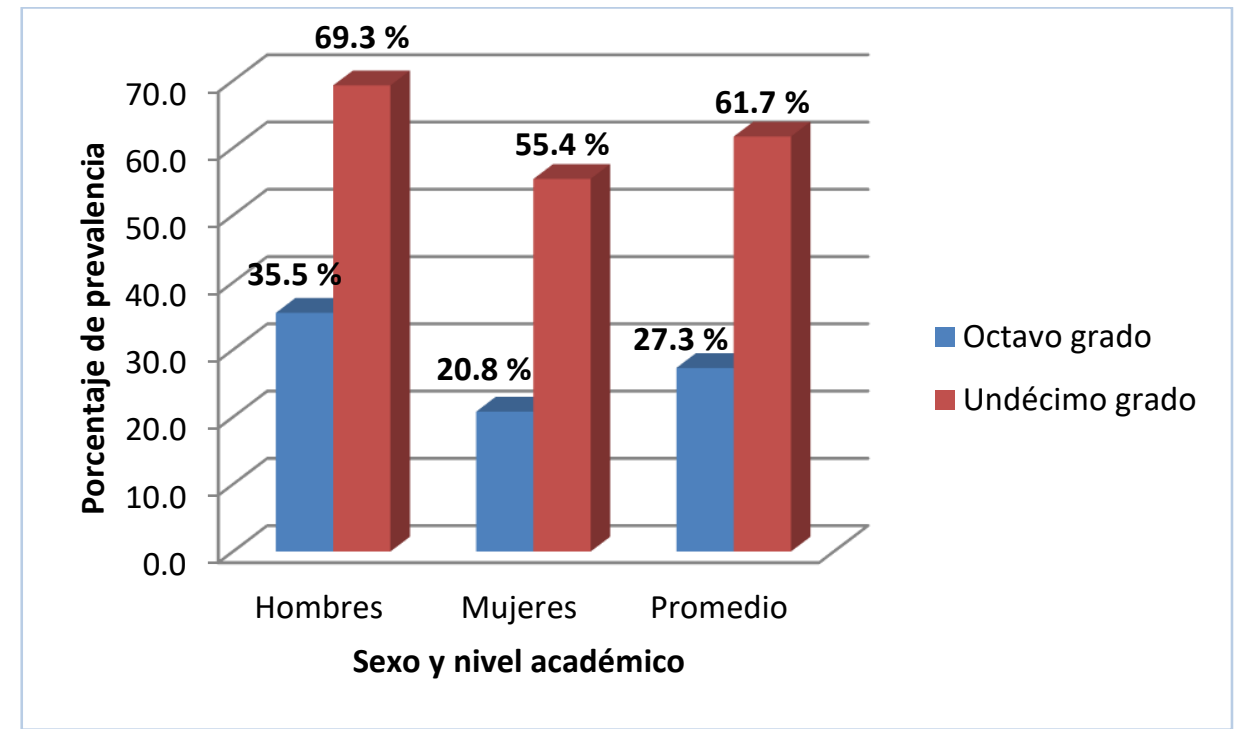

Fuente: Elaborada por los autores. 
A nivel nacional, Mc Donald et al. (2015), para alumnos de octavo grado, reporta un promedio de prevalencia de consumo de bebidas alcohólicas de $11,7 \%$, siendo ligeramente superior en estudiantes de sexo masculino $(11,8 \%)$ que en estudiantes de sexo femenino (11,6\%). También señala que la región con mayor prevalencia de consumo de bebidas alcohólicas a esta edad es la provincia de Los Santos con un $27,5 \%$, cifra similar a la encontrada en el presente estudio (27,3\%).

Referente a la edad promedio del primer consumo, se obtiene valores de 12,1 años y 14,3 años en octavo grado y undécimo grado, respectivamente. Estos resultados concuerdan con lo reportado en otras investigaciones, donde la edad promedio del primer consumo se localiza entre 12 a 15 años (Espada et al., 2000; López, González y Pacheco, 2006; Caneto, Vera, Pautassi y Pilatti, 2015; Morales- González et al., 2015). Cabe destacar que, en promedio, los hombres inician primero el consumo de bebidas alcohólicas. En este sentido, en octavo grado la edad promedio del primer consumo fue 12,10 años en hombres y 12,14 años en mujeres, mientras que en undécimo grado fue 14 años en los hombres y 14, 62 años en las mujeres (figura 2).

Figura 2. Edad promedio del primer consumo de bebidas alcohólicas en estudiantes del distrito de Las Tablas, por sexo y nivel académico.

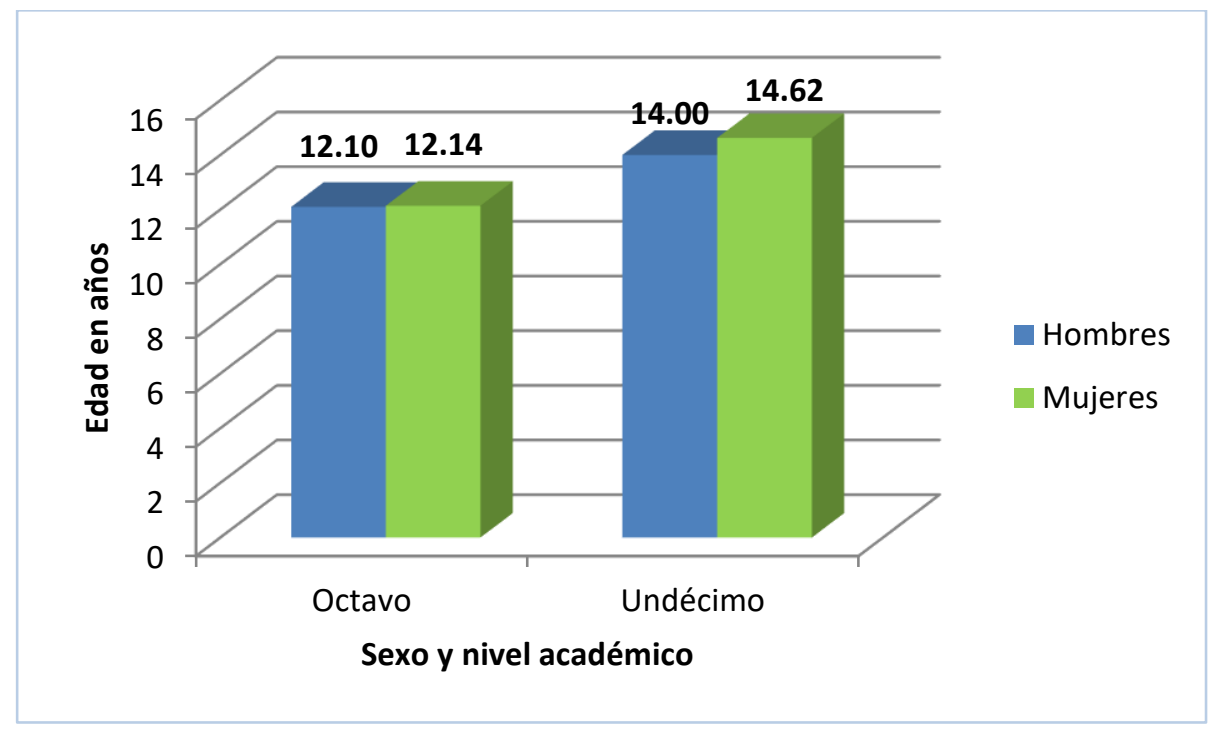

Fuente: Elaborada por los autores. 
Preocupa el descenso en la edad promedio del primer consumo en estudiantes de octavo grado, en comparación con undécimo grado (tabla 1), máxime cuando en los primeros, $3(8,6 \%)$ estudiantes reportan una edad de inicio de consumo de alcohol a los siete-ocho años y tres $(8,6 \%)$ a los $9-10$ años. Estos reportes concuerdan con los obtenidos por Cicua, Méndez y Muñoz (2008), donde registra una edad de inicio desde los seis y siete años, en estudiantes de 12 a 17 años, en la ciudad de Bogotá (Colombia). En contraposición, en undécimo grado, tres (3\%) de los estudiantes que han iniciado el consumo de alcohol, lo han hecho a los 10 años o antes.

Tabla 1. Estadísticas descriptivas de la edad promedio del primer consumo de bebidas alcohólicas, en estudiantes muestreados del Colegio Manuel María Tejada Roca, 2018.

\begin{tabular}{l|r|r}
\hline Media & Octavo grado & Undécimo grado \\
\hline Varianza & 12.1142857 & 14.3131313 \\
\hline Observaciones & 3.57478992 & 4.05400948 \\
\hline Mínimo & 35 & 99 \\
\hline Máximo & 7 & 3 \\
\hline Grados de libertad & 14 & 17 \\
\hline F & 132 & \\
\hline Probabilidad & 31.8076744 & \\
\hline Valor crítico de F & $9.9083 \mathrm{E}-08$ & \\
\hline
\end{tabular}

Fuente: Datos generados por los autores.

Al comparar la edad promedio del primer consumo, se obtiene que la $\mathrm{F}$ calculada $(31,8076744)$ es mayor que el valor crítico de $F(3,91287503)$ y que la probabilidad de estadística 9,9083E-08 es inferior a 0,05, por lo que se asume que existen diferencias significativas en la edad promedio del primer consumo entre los estudiantes muestreados, por nivel académico (tabla 1). Se consideran entre los principales factores que agudizan esta problemática, la cultura de considerarse el alcohol un recurso de convivencia social (Domínguez, 2017; Torrijos, 2019), el elevado número de fiestas de calle que existen en la provincia y la publicidad constante del producto (D. M. González, comunicación personal, 3 de septiembre de 2018), así como el poco control por parte de padres o tutores legales. 
Por otro lado, la curiosidad (56,7 \%) se reporta como el principal factor que motivó a los jóvenes a consumir alcohol por primera vez (figura 3), concordando con lo reportado en otras investigaciones (Moral, Rodríguez y Sirvent, 2005; Cabrera-Jiménez et al., 2013). Especulamos que esta situación se debe a que los jóvenes están rodeados de adultos que consumen bebidas alcohólicas sin ningún tipo de restricción, dando como resultado que los jóvenes, desde niños, se mentalicen que el consumo de bebidas alcohólicas es una actividad normal. Esto se ve reforzado en las fiestas, incluyendo las fiestas para bebés y niños, donde no dejan de faltar las bebidas alcohólicas, lo que de paso concuerda con nuestra segunda causa de consumo temprano, la cual es para celebrar algo $(34,1 \%)$.

\section{Figura 3. Factores motivacionales asociados al primer consumo de bebidas alcohólicas} en estudiantes del distrito de Las Tablas.

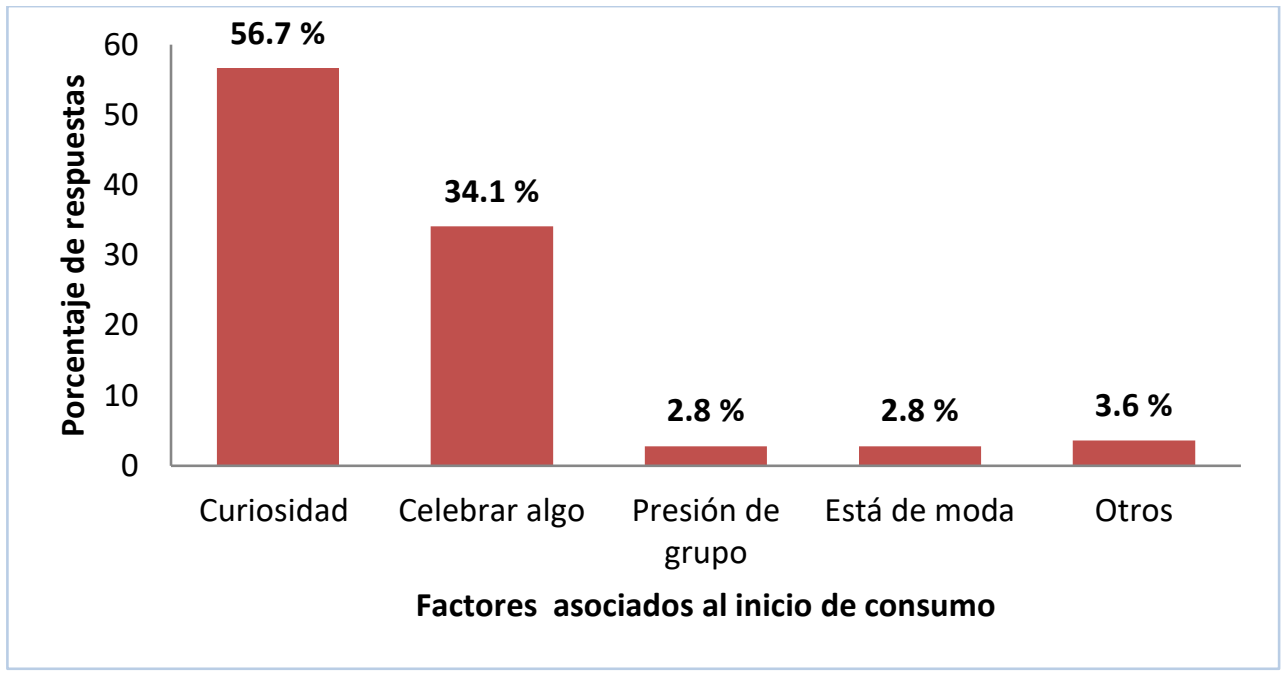

Fuente: Elaborada por los autores.

En relación al tipo de bebida alcohólica consumida por los estudiantes, las cervezas $(42,6 \%)$ ocupan el primer lugar (figura 4), coincidiendo con lo obtenido en otros estudios (Cicua et al., 2008; Andrade, Yepes y Sabogal, 2013; Cabrera - Jiménez et al., 2013; Morales - González et al., 2015; Tegoma-Ruiz y Cortaza-Ramírez, 2016). Esto obedece a que las cervezas son bebidas alcohólicas de fácil adquisición, con amplia publicidad y vendidas a bajos precios en multitud de comercios. Además, son consideradas un trago suave (con bajo porcentaje de alcohol) y comercializadas en varios tamaños. 
Figura 4. Bebidas alcohólicas preferidas por estudiantes del distrito de Las Tablas.

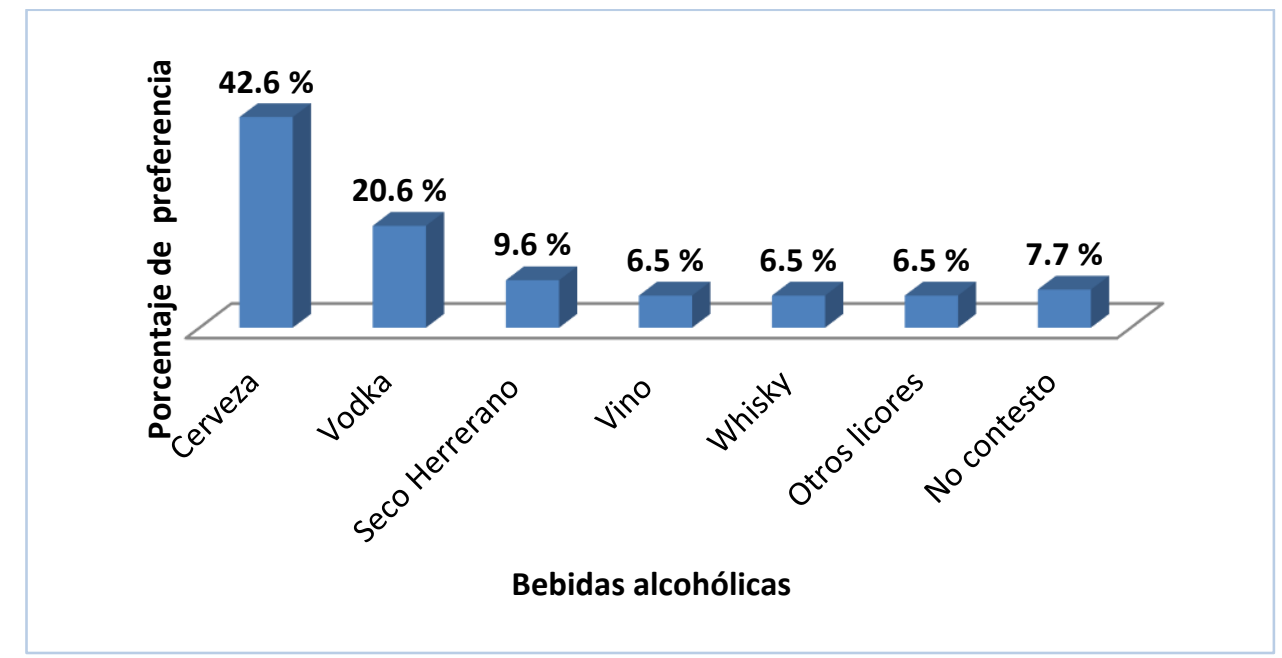

Fuente: Elaborada por los autores.

Referente a la a prueba AUDIT, la sub- muestra estuvo conformada por 104 estudiantes (54 del sexo masculino y 50 del sexo femenino). La mayoría de los jóvenes (55,8 $\%)$, se localizan como consumo de bajo riesgo. Sin embargo, preocupa que otra gran parte, se localicen dentro del estándar consumo riesgoso (37,5 \%), y peor aún, que encontremos cuatro $(3,8 \%)$ estudiantes con consumo perjudicial y tres $(2,9 \%)$ estudiantes con dependencia al alcohol (figura 5).

Figura 5. Riesgo de los alumnos que consumen actualmente bebidas alcohólicas, por sexo, de acuerdo al Test AUDIT.

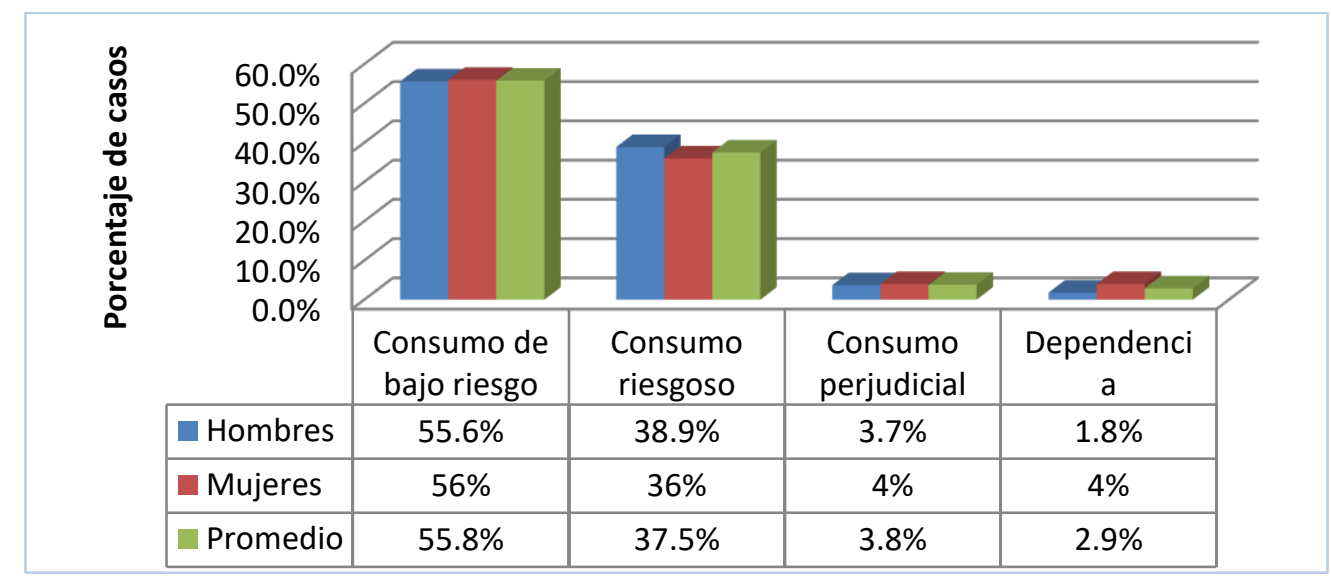

Fuente: Elaborada por los autores. 
Estos resultados, grosso modo, concuerdan con lo obtenido en investigaciones realizadas en otras regiones (Tamez-Rodríguez et al., 2013; Morales- González et al., 2015). No obstante, es preocupante que, un gran porcentaje de estudiantes que consumen bebidas alcohólicas, presentan consumo riesgoso (38,9 \% de varones y $36 \%$ de mujeres), especialmente al considerar el hecho de que, en la sociedad, en la que se desenvuelven, existen pocas restricciones sociales al consumo de alcohol, tener acceso a bebidas alcohólicas es muy sencillo y constantemente se realizan fiestas de calle y celebraciones.

En base a los resultados de la prueba AUDIT y a las características de la región en estudio, urge implementar programas preventivos al uso y abuso de bebidas alcohólicas por los estudiantes, desde el nivel primario, ya que es alarmante que en octavo grado, se registren dos (4\%) mujeres con consumo perjudicial y una (2\%) con dependencia al alcohol. Por consiguiente, se necesitan programas de prevención e intervención, que busquen desarrollar en los adolescentes la habilidad para rechazar ofrecimientos de bebidas alcohólicas y poder divertirse sin la necesidad de consumir dicho producto. Asimismo, al estar prohibido el suministro o expendio de bebidas alcohólicas a menores de edad, desde 1973 (Ley 55, 1973, art. 25), las autoridades competentes y tutores legales del menor deben estar vigilantes a esta prohibición, ya que muchos adolescentes desconocen, o hacen caso omiso a esta normativa, no reparando en los riesgos a los que pueden exponerse al consumir regularmente bebidas alcohólicas.

Finalmente, para verificar si existen diferencias significativas entre el rendimiento académico de estudiantes que consumen bebidas alcohólicas versus aquellos que no lo hacen, se realizó un Análisis de varianza de un factor del promedio de notas. En este sentido, al obtenerse que la F calculada $(15,7140763)$ es mayor al valor crítico de $F(3,874410219)$ y que la probabilidad de estadístico 9,3286E-05 es inferior a 0,05, se asume que existen diferencias significativas entre ambos grupos (tabla 2). Estos resultados son consistentes con los hallazgos de otros estudios realizados en adolescentes, donde hacen referencia al consumo de bebidas alcohólicas como una de las causales de bajo rendimiento académico (Caso - Niebla et al., 2007; Lituma - Aguirre et al., 2013; Morales - González et al., 2015). 
Tabla 2. Estadísticas descriptivas del promedio de notas de los estudiantes muestreados en el colegio Manuel María Tejada Roca, durante el primer trimestre de 2018.

\begin{tabular}{l|r|r}
\hline & Han Bebido & No han bebido \\
\hline Media & 3.75163934 & 3.93597561 \\
\hline Varianza & 0.16350969 & 0.14219475 \\
\hline Observaciones & 122 & 164 \\
\hline Mínimo & 2.9 & 3.0 \\
\hline Máximo & 4.7 & 4.8 \\
\hline Grados de libertad & 284 & \\
\hline F & 15.7140763 & \\
\hline Probabilidad & $9.3286 \mathrm{E}-05$ & \\
\hline Valor crítico de F & 3.874410219 & \\
\hline
\end{tabular}

Fuente: Datos generados por los autores.

\section{Conclusiones}

- La prevalencia en el consumo de bebidas alcohólicas en octavo grado fue $27,3 \%$, mientras que en undécimo grado se incrementa hasta el $61,7 \%$, registrándose una mayor prevalencia en estudiantes varones.

- Al registrarse una reducción significativa en la edad promedio del primer consumo, en estudiantes de octavo grado, con relación a los estudiantes de undécimo grado, urge implementar programas que contribuyan a la prevención del consumo de bebidas alcohólicas, en niños y adolescentes.

- La curiosidad es el principal factor asociado al primer consumo de bebidas alcohólicas en estudiantes, por lo que resulta vital la disminución de campañas publicitarias alusivas al producto en comunidades y medios de comunicación.

- Las cervezas son las bebidas alcohólicas de mayor preferencia por los estudiantes, debido a su diversidad, fácil adquisición, amplia publicidad, y bajo precio.

- Los resultados de la prueba AUDIT indican que de los estudiantes que actualmente consumen bebidas alcohólicas, 55,8 \% presentan consumo de bajo riesgo, 37,5 \% consumo riesgoso, 3,8 \% consumo perjudicial y 2,9 \% dependencia al alcohol, lo que implica que las autoridades y tutores legales deben participar activamente en prevenir que el adolescente desarrolle dependencia a las bebidas alcohólicas. 
- El consumo de bebidas alcohólicas por los estudiantes afecta negativa y significativamente su rendimiento académico, por lo que es necesario una mayor fiscalización de la accesibilidad de los mismos a estos productos.

\section{Referencias bibliográficas}

Aguilar-Barojas, S. (2005). Fórmulas para el cálculo de la muestra en investigaciones de salud. Salud en Tabasco 11 (1-2): 333-338. Recuperado de: http://www.redalyc.org/articulo.oa?id=48711206

Andrade S, J.A., Yepes T, A. J., y Sabogal G., M. L. (2013). Resistencia a la presión de grupo y consumo de alcohol en 50 jóvenes universitarios entre los 16 y 22 años de la universidad de San Buenaventura Medellín, extensión Ibagué. Revista Colombiana de Ciencias Sociales 4 (1): 12-23. Recuperado de: http://www.funlam.edu.co/uploads/proyectotoma/5 Resistencia a la presion de grupo.pdf

Babor, T.F., Higgins-Biddle, J.C., Saunders, J.B., y Monteiro, M. G. (2001). Cuestionario de Identificación de los trastornos debidos al consumo de alcohol: pautas para su utilización en atención primaria. Organización Mundial de la salud (WHO/MSD/MSB/01.6a). Recuperado de: https://www.who.int/substance abuse/activities/en/AUDITmanualSpanish.pdf

Cabrera-Jiménez, M., López- Molina, J., González-Flores, J. E., Hernández - Acevedo, A. G., Estrella-López, L. R., Hernández-Ruiz, H.,...y Martínez- Tornero, Z. Z. (2013). Consumo de alcohol y factores de riesgo coadyuvantes en los habitantes de una colonia de Iztapalapa, Distrito Federal, año 2013. VERTIENTES 16 (2), 83-89. Recuperado de: http://www.revistas.unam.mx/index.php/vertientes/article/view/51708

Casas, K. T. y Parrado C, F. (2013). Consumo de alcohol y conducta social en hombres adolescentes universitarios: un estudio observacional. Cultura, Educación y Sociedad 4(1), 89-98. Recuperado de: https://n9.cl/ljot 
Caneto, F., Vera, B. del V., Pautassi, R. M., y Pilatti, A. (2015)._Personalidad, edad de inicio y problemas por consumo de alcohol en estudiantes. Quaderns de Psicologia,_17(2), 1935. Recuperado de: http://dx.doi.org/10.5565/rev/qpsicologia.1247

Caso- Niebla, J. y Hernández- Guzmán, L. (2007). Variables que inciden en el rendimiento académico de adolescentes mexicanos. Revista Latinoamericana de Psicología 39 (3), 487-501. Recuperado de: http://www.redalyc.org/pdf/805/80539304.pdf

Cicua D., Méndez, M., y Muñoz L. (2008). Factores en el consumo de alcohol en adolescentes. Pensamiento psicológico 4 (1), 123-12. Recuperado de: http://www.redalyc.org/articulo.oa?id=80111671008

Chacón, J. A. (15 de febrero de 2013). El alcohol hace fiesta con los jóvenes panameños. Panamá América. Recuperado de: http://www.panamaamerica.com.pa/nacion/elalcohol-hace-fiesta-con-los-jovenes-panamenos-863299

Consejo Nacional de Legislación. (10 de julio de 1973). Por la cual se regula la administración, fiscalización y cobro de varios tributos municipales. Ley 55 de 1973. Gaceta Oficial $\quad N^{\circ}$ 17397. Recuperado de: https://docs.panama.justia.com/federales/leyes/55-de-1973-jul-26-1973.pdf

Delgado, B., Bautista, R., Inglés, C. J., Espada, J. P., Torregrosa, M. S., y García-Fernández, J. M. (2005). Diferencias de género en el consumo de alcohol y tabaco de estudiantes de educación secundaria obligatoria. Salud y drogas 5 (2), 55-65. Recuperado de: http://www.redalyc.org/pdf/839/83905203.pdf

Delgado, J. R. P., Flores, M. I. B., y Palos, P. A. (2007). Consumo de alcohol y conducta sexual de riesgo en adolescentes. Psychology International 18 (4), 1-13. Recuperado de: https://www.apa.org/international/pi/2007/11/consumo.pdf

Domínguez, Y. (10 de julio de 2017). Jóvenes le meten duro al "guaro". Día a Día. Recuperado de: https://www.diaadia.com.pa/el-pais/jovenes-le-meten-duro-alguaro-326002

Espada S, J. P., Méndez C, F. X., e Hidalgo M, M. D. (2000). Consumo de alcohol en escolares: descenso de la edad de inicio y cambios en los patrones de ingesta. Adicciones 12 (1), 57-64. Recuperado de: https://n9.cl/3489k 
Flores H., J. (2016). Factores de riesgo asociados al consumo de bebidas alcohólicas en los adolescentes de dos instituciones educativas una pública y una privada (tesis de pregado). Universidad Ricardo Palma, Lima, Perú. Recuperado de: http://repositorio.urp.edu.pe/handle/urp/752

Landero H, R. y Villarreal G, M.E. (2007). Consumo de alcohol en estudiantes en relación con el consumo familiar y de los amigos. Psicología y Salud 17 (1), 17- $23 . \quad$ Recuperado de: https://www.uv.es/lisis/m-villarreal/consum-alcohol2007.pdf

Lituma - Aguirre, D., Abanto- Hinestroza, R.J., Altamirano-Gomes, J.C., Cahuana-Castillo, F., Castañeda- Alarcón, D., Leiva- Valentin, R.G.,... y Ríos- Zeballos, W. (2013). Factores asociados a bajo rendimiento académico en un colegio estatal de la localidad de Armatambo Chorrillos. Revista de la Facultad de Medicina Humana de la Universidad Ricardo Palma (2): 21-25. Recuperado de: https://n9.cl/bkof

López-Cisneros, M. A., Villar - Luis, M. A., Alonso- Castillo, M. M., Alonso - Castillo, M. T. de J., y Rodríguez- Aguilar, L. (2013). Actitud ante el consumo y no consumo de alcohol en estudiantes de preparatoria - México. Revista da Escola de Enfermagem da USP 47 (4), 815-821. Recuperado de: http://www.scielo.br/pdf/reeusp/v47n4/0080-6234reeusp-47-4-0815.pdf

López, L., González, L., y Pacheco, L. (2006). Prevalencia del consumo de bebidas alcohólicas en estudiantes de colegios de Asunción. Recuperado de: http://scielo.iics.una.py/pdf/anales/v38n3/v38n3a13.pdf

McDonald, P., Anselmo, J., Moreno R, A. L., de Bradshaw, A., Cumbrera O, A., Bradshaw M, R., y Mendoza, E. (2015) Prevalencia de la depresión y tipos de maltrato en estudiantes de VIII grado. Escuelas públicas de distritos cabecera de provincias y comarcas. Instituto Conmemorativo Gorgas de Estudios de la Salud. República de Panamá. Recuperado de: https://n9.cl/ikp6

Moniño G, M., Piñero R, E., Arense G. J., y Cerezo R., F. (2013). Violencia escolar y consumo de alcohol y tabaco en estudiantes de Educación Secundaria. European Journal of Investigation in Health, Psychology and Education 3 (2), 137-14. Recuperado de: https://dialnet.unirioja.es/servlet/articulo?codigo=4519062 
Moral J, M. V., Rodríguez D, F. J., y Sirvent, R. (2005). Motivadores de consumo de alcohol en adolescentes: análisis de diferencias inter-género y propuesta de un continuum etiológico. ADICCIONES 17 (2): 105-120. Recuperado de: http://www.redalyc.org/pdf/2891/289122003003.pdf

Morales-González, F., Cabrera-Jiménez, M., Pérez-Barajas, C.J., y Amaro-Franco, M. L. (2015). El consumo de alcohol en adolescentes de una secundaria de Ciudad Nezahualcóyotl Frecuencia y características sociales, 2015. VERTIENTES 18 (1), 8-15. Recuperado de: https://n9.cl/woo2

Pastor S., P. (2002). Percepción del alcohol entre los jóvenes. ADICCIONES 14 (1), 123- 131 Recuperado de: http://www.adicciones.es/index.php/adicciones/article/view/522

Simmons, R. (03 de enero de 2016). Panamá, entre los países más bebedores de alcohol de la región. La Prensa. Recuperado de: https://www.prensa.com/sociedad/Altoconsumo alcohol 0 4383311741.html

Tamez-Rodríguez, V. A., Cerda-Flores, R. M., Marín-Peña, E., Machado-Cervantes, J.M., Dávila-Canales, R., Rodríguez-Aguilar, L., y Martínez-Maldonado, R. (2013). Evaluación del consumo de alcohol y sus motivaciones en_estudiantes del noreste de México. Medicina Universitaria 15 (58), 3-9. Recuperado de: https://n9.cl/k919

Tegoma-Ruiz, V. M., y Cortaza-Ramírez, L. (2016). Prevalencia del consumo de alcohol en adolescentes de una secundaria de Coatzacoalcos, Veracruz. Enfermería Universitaria 13 (4), 239-245. Recuperado de: http://www.scielo.org.mx/pdf/eu/v13n4/23958421-eu-13-04-00239.pdf

Téllez M, J., y Cote M, M. (2006). Alcohol etílico: Un tóxico de alto riesgo para la salud humana socialmente aceptado. Rev Fac Med Univ Nac Colomb 54 (1): 32-47. Recuperado de: http://www.scielo.org.co/pdf/rfmun/v54n1/v54n1a05.pdf

Torrijos L, J. M. (19 de junio de 2019). Consumo excesivo de alcohol: las complejidades al afrontar un problema multidimensional. Panamá América. Recuperado de: https://www.panamaamerica.com.pa/sociedad/consumo-excesivo-de-alcohol-lascomplejidades-al-afrontar-un-problema-multidimensional-0 
51. Visión Antataura, Vol.4, No.1, Junio - Noviembre 2020

\section{ANEXO 1}

Figura 6. Fórmula utilizada para calcular tamaño significativo de muestra

$$
n=\frac{Z_{\alpha}^{2} N p q}{e^{2}(N-1)+Z_{\alpha}^{2} p q}
$$

Fuente: Aguilar-Barojas, 2005 